\title{
Estrogen Intake and Copper Depositions: Implications for Alzheimer's Disease?
}

\author{
Florian Amtage $^{a} \quad$ Dzelila Birnbaum $^{d}$ Thomas Reinhard $^{d}$ \\ Wolf-Dirk Niesen $^{a} \quad$ Cornelius Weiller ${ }^{a} \quad$ Irina Mader $^{b}$ \\ Philipp T. Meyer ${ }^{c} \quad$ Michel Rijntjes $^{a}$ \\ Departments of ${ }^{a}$ Neurology, ${ }^{b}$ Neuroradiology and ${ }^{c}$ Nuclear Medicine, University Medical \\ Center Freiburg, and ${ }^{\mathrm{d}}$ University Eye Hospital Freiburg, Freiburg, Germany
}

\section{Key Words}

$\left[{ }^{11} \mathrm{C}\right]$ PIB PET $\cdot$ Alzheimer's disease $\cdot$ Ceruloplasmin $\cdot$ Copper $\cdot$ Estrogen

\begin{abstract}
We present a patient with chronic postmenopausal estrogen intake with presence of KayserFleischer ring in the cornea and Alzheimer's disease and discuss the pathophysiological mechanisms of estrogen intake and copper accumulation in various tissues, including the central nervous system. Sonography was compatible with copper accumulation in the basal ganglia, but the patient showed no clinical signs of Wilson's disease. Magnetic resonance imaging and positron emission tomography revealed a typical pattern for Alzheimer's disease. We propose increased copper levels as a direct effect of estrogen intake due to an augmented ATP7A-mRNA in the intestine. Moreover, we discuss the impact of elevated free serum copper on accompanying Alzheimer's disease, knowing that copper plays a crucial role in the formation of amyloid plaques and tau aggregation. This might offer a partial explanation for the observation that postmenopausal estrogen therapy is associated with a higher risk of mild cognitive impairment and Alzheimer's disease.

(c) 2014 S. Karger AG, Basel
\end{abstract}

\section{Introduction}

Copper deposition in the Descemet membrane of the cornea, known as Kayser-Fleischer ring, is a rare finding in daily practice. If present, it is usually assumed to be pathognomonic for Wilson's disease (WD; a hepatolenticular degeneration and autosomal recessive movement disorder), but a Kayser-Fleischer ring is also rarely seen in other diseases, mainly

Florian Amtage, MD

Department of Neurology, University Medical Center Freiburg

Breisacherstrasse 64

DE-79106 Freiburg (Germany)

E-Mail florian.amtage@uniklinik-freiburg.de 
Amtage et al.: Estrogen Intake and Copper Depositions: Implications for Alzheimer's Disease?

of hepatic or hematological origin, indicating an imbalance of copper homeostasis $[1,2]$. Copper is virtually totally incorporated into ceruloplasmin while small amounts are bound to serum albumin. In WD, ceruloplasmin deficiency leads to copper depositions in the cornea and brain via a consecutive rise of free serum copper. Frequently, a Kayser-Fleischer ring can be detected prior to the appearance of neurological symptoms [3] and an efficient diagnosis and treatment of copper accumulation may prevent the development of the movement disorder.

Contraceptives with estrogenic effects are known to elevate serum copper and ceruloplasmin levels $[4,5]$. The underlying pathomechanism is not fully understood, but several authors propose an increase of ceruloplasmin as a direct effect of estrogen and, consequently, a rise of total copper content in the serum $[4,5]$. Whatever the mechanism, the alteration of copper homeostasis is not listed in the drug information of oral contraceptives. However, in the last years, 3 cases with corneal deposition due to chronic intake of contraceptives have been reported, showing that changes in serum copper can be of clinical importance $[6,7]$.

\section{Case Report}

A 68-year-old woman with a 4-year history of Alzheimer's disease (AD) was admitted to the University Eye Hospital in Freiburg, Germany, for surgery of a bilateral pterygium and cataract. Preinterventional slit-lamp examination revealed a yellow-greenish deposition in the posterior corneal stroma and the Descemet membrane at the limbus of both corneas (fig. $1 \mathrm{a}, \mathrm{b})$. Urine copper (58.5 $\mu \mathrm{g} / 24 \mathrm{~h})$, serum copper $(106 \mu \mathrm{g} / \mathrm{dl})$ and ceruloplasmin $(38 \mathrm{mg} / \mathrm{dl})$ levels as well as transaminases and bilirubin were within the normal ranges. Neurological examination showed a slight postural tremor of both upper limbs and the head. The tremor syndrome had been present since the patient's adolescence and, in the light of a positive family history, it was judged to be an essential tremor. Neither dystonic, atactic or bulbar symptoms nor extrapyramidal signs including rigidity were present. Thus, the diagnosis of WD could not be made. Due to progressive AD ('probable AD' according to NINCDS-ADRDA criteria), anomia and apraxia were present. The cognitive state of the patient was evaluated by Mini-Mental State Examination, where she reached 10 of 30 points, with 4 points for orientation (time, town, country, district), all 3 points for the registration of 3 words, 1 point for language (name a pencil) and 2 points for the 3-stage command; all speaking for a moderate to severe impairment. The patient reported a daily 2-mg postmenopausal hormone estradiol intake over the past 22 years until one year before.

Magnetic resonance imaging (MRI) revealed brain atrophy of the temporal and parietal lobes (fig. 1c). Susceptibility-weighted MRI showed no copper accumulation in the basal ganglia. Neurosonography of the midbrain and basal ganglia displayed a right accentuated hyperechogenicity of both lentiform nuclei (fig. 1d). In line with the clinical diagnosis, positron emission tomography (PET) studies revealed typical features of AD (fig. 2): [ $\left.{ }^{18} \mathrm{~F}\right]$ fluorodeoxyglucose (18FDG) PET demonstrated a pronounced bilateral temporoparietal hypometabolism, while ${ }^{11} \mathrm{C}$-labeled Pittsburgh Compound-B ([11C]PIB) PET showed strong binding to cortical fibrillar amyloid- $\beta$ depositions in an AD-typical distribution.

\section{Discussion}

The enzyme ATP7B facilitates the transfer of copper into the Golgi apparatus of hepatocytes, where copper is incorporated into ceruloplasmin. In WD, a mutation of the copper- 
Amtage et al.: Estrogen Intake and Copper Depositions: Implications for Alzheimer's Disease?

transporting gene ATP7B leads to secondary ceruloplasmin deficiency with a reduced halflife. Free serum copper accumulates in tissues like the eye (Kayser-Fleischer ring, sunflower cataract), brain (mainly basal ganglia), kidney and liver. A Kayser-Fleischer ring can be detected in approximately $60 \%$ of presymptomatic patients with WD, whereas virtually every patient with WD and neurological symptoms presents corneal copper deposition [3, 8]. Furthermore, a Kayser-Fleischer ring has been reported in other hepatic and hematological diseases $[1,2]$. In the latter cases, elevated serum copper level was detected, whereas ceruloplasmin remained normal. Therefore, a relative surplus of free serum copper seems to be the common factor for tissue accumulation, rather than the absolute amount of copper or ceruloplasmin.

An increase of ceruloplasmin and serum copper due to chronic estrogen administration has been known since 1966 [4,5]. Moreover, both serum copper and ceruloplasmin are elevated during pregnancy [9], a physiologic state of raised estrogen levels. Several authors suggest an estrogen-induced increase of ceruloplasmin and, secondary to that, a rise of the amount of total copper $[4,5]$. An explanation for a missing clinical manifestation of these increased serum copper levels might be that copper is incorporated into ceruloplasmin, preventing the accumulation of free serum copper. However, the occurrence of copper depositions in the cornea due to long-lasting estrogen intake argues for a direct impact of estrogens on copper uptake, since ceruloplasmin remains normal in these cases [6, 7].

Hardman et al. [10] found evidence that estrogen has a direct impact on the ATP7A transporter of human placental cells, leading to a stimulation of the copper transport via an increase of ATP7A-mRNA expression. Transferring these data to enterocytes, where ATP7A plays a crucial role for the regulation of copper uptake [11], estrogen could upregulate ATP7A-mRNA, inducing an increased copper uptake via the intestine. This seems plausible, since an adequate copper supply to the embryo under physiologic conditions must be on the basis of an elevated intestinal copper uptake of the expectant mother. Therefore, we propose that the increase of ceruloplasmin after estrogen treatment is secondary to an enhanced copper uptake. However, in patients with a Kayser-Fleischer ring under estrogen therapy, this hepatic compensatory mechanism seems to be disturbed, possibly due to having a defect on the ceruloplasmin gene or being heterocygotes for WD. New findings suggest an association of an ATP7B polymorphism and sporadic AD which might be of interest in this context, since this polymorphism results in a disturbed function of the copper transporter, leading to an increased level of free serum copper [12]. Since in our case estradiol therapy was discontinued one year ago, ceruloplasmin and serum copper were at normal levels. We did not restart estradiol therapy to prove the pathomechanism because of ethical considerations mentioned below. However, in the 3 cases previously described, the causality between estrogen intake and enhanced serum copper has been described [6, 7].

In WD, copper accumulation can be diagnosed by basal ganglia sonography, showing hyperechogenicity of the lentiform nucleus [13]. In our patient, neurosonography of the basal ganglia revealed a right accentuated hyperechogenicity of both lentiform nuclei, supporting copper deposition in the basal ganglia, without presenting neurological symptoms compatible with WD. Walter et al. [13] also found this hyperechogenicity in 2 of 3 presymptomatic patients with WD. This could be explained by the time lag ranging from 7 to 37 years between copper accumulation (with secondary neurodegeneration due to oxidative stress) and overt neurological symptoms [8]. Susceptibility-weighted MRI showed no incidence of copper accumulation in the brain, but abnormal MRI findings in presymptomatic WD seem to be rare [3].

The role of copper in the pathogenesis of $\mathrm{AD}$ is still controversial, but there is increasing evidence that besides iron and zinc, free serum copper is involved in the aggregation of 
amyloid- $\beta$-forming amyloid plaques [14]. Moreover, a meta-analysis confirmed increased serum copper levels in patients with AD [15]. Copper is a redox-active metal and plays an important role as a catalyst in the production of reactive oxygen species. Furthermore, copper is assumed to build complexes with tau protein [16], the second pathologic hallmark of AD. It seems noteworthy that the copper transporter ATP7A is present in the brain. A corelease of amyloid- $\beta$ and copper into the synaptic cleft increases the probability of building a redox-active amyloid- $\beta$-copper complex leading to oxidative stress, which enhances the forming of amyloid plaques. Estrogen therapy could have an impact on the expression of the ATP7A transporter in the brain as well, but this has not been demonstrated yet.

Although a case report like ours does not necessarily provide evidence for causal relationships, it allows, nevertheless, formulating hypotheses for further investigations and research. Certainly, it cannot be proven that the occurrence of AD in the present case is due to estrogen therapy. However, it is very tempting to assume a causal role after more than 20 years of estradiol intake leading to a Kayser-Fleischer ring and presumed copper depositions in the basal ganglia and given the crucial role of copper in the pathogenesis of AD pathology. Furthermore, this hypothesis might offer a partial explanation for the observation that postmenopausal estrogen therapy is associated with a higher risk of mild cognitive impairment, which is commonly the first stage of $\mathrm{AD}$ [17].

In conclusion, we suggest that augmented ATP7A-mRNA in the intestine as a direct effect of estrogen therapy facilitates the absorption of copper, leading to increased serum copper levels. In some patients, hepatic function might be unable to cope with an increased copper load, resulting in corneal copper depositions and cerebral copper accumulation in the basal ganglia. Furthermore, similar to findings in WD patients, we demonstrate hyperechogenicity of both lentiform nuclei, indicating cerebral copper accumulation in the basal ganglia in our patient. Increased levels of free serum copper might have an impact on $\mathrm{AD}$, since the same ATP7A transporter for copper homeostasis is present in the brain and copper plays a crucial role in oxidative stress, the formation of amyloid plaques and tau aggregation. Although we cannot provide evidence for causality in the present case, we want to stimulate further investigations on the impact of estrogen on copper homeostasis and the link between serum copper and the pathophysiology of $\mathrm{AD}$, since this might have consequences for monitoring hepatic regulatory mechanisms in patients receiving estrogens.

\section{Disclosure Statement}

The authors have no relevant financial disclosures or conflict of interests to declare.

\section{References}

1 Aldave AJ, King JA, Kim BT, Hopp L: Corneal copper deposition associated with chronic lymphocytic leukemia. Am J Ophthalmol 2006;142:174-176.

2 Tauber J, Steinert RF: Pseudo-Kayser-Fleischer ring of the cornea associated with non-Wilsonian liver disease. A case report and literature review. Cornea 1993;12:74-77.

-3 Taly AB, Meenakshi-Sundaram S, Sinha S, Swamy HS, Arunodaya GR: Wilson disease: description of 282 patients evaluated over 3 decades. Medicine (Baltimore) 2007;86:112-121.

-4 Carruthers ME, Hobbs CB, Warren RL: Raised serum copper and caeruloplasmin levels in subjects taking oral contraceptives. J Clin Pathol 1966;19:498-500.

5 O'Leary JA, Spellacy WN: Serum copper alteration after ingestion of an oral contraceptive. Science 1968;162:682.

6 Garmizo G, Frauens BJ: Corneal copper deposition secondary to oral contraceptives. Optom Vis Sci 2008;85:E802-E807. 
Amtage et al.: Estrogen Intake and Copper Depositions: Implications for Alzheimer's Disease?

7 Orlin A, Orlin SE, Makar GA, Bunya VY: Presumed corneal copper deposition and oral contraceptive use. Cornea 2010;29:476-478.

8 Machado A, Chien HF, Deguti MM, Cancado E, Azevedo RS, Scaff M, Barbosa ER: Neurological manifestations in Wilson's disease: report of 119 cases. Mov Disord 2006;21:2192-2196.

-9 Louro MO, Cocho JA, Tutor JC: Assessment of copper status in pregnancy by means of determining the specific oxidase activity of ceruloplasmin. Clin Chim Acta 2001;312:123-127.

10 Hardman B, Michalczyk A, Greenough M, Camakaris J, Mercer JF, Ackland ML: Hormonal regulation of the Menkes and Wilson copper-transporting ATPases in human placental Jeg-3 cells. Biochem J 2007;402:241250.

11 van den Berghe PV, Klomp LW: New developments in the regulation of intestinal copper absorption. Nutr Rev 2009;67:658-672.

12 Bucossi S, Mariani S, Ventriglia M, Polimanti R, Gennarelli M, Bonvicini C, Pasqualetti P, Scrascia F, Migliore S, Vernieri F, Rossini PM, Squitti R: Association between the c. 2495 A>G ATP7B polymorphism and sporadic Alzheimer's disease. Int J Alzheimers Dis 2011;2011:973692.

13 Walter U, Krolikowski K, Tarnacka B, Benecke R, Czlonkowska A, Dressler D: Sonographic detection of basal ganglia lesions in asymptomatic and symptomatic Wilson disease. Neurology 2005;64:1726-1732.

14 Bucossi S, Ventriglia M, Panetta V, Salustri C, Pasqualetti P, Mariani S, Siotto M, Rossini PM, Squitti R: Copper in Alzheimer's disease: a meta-analysis of serum,plasma, and cerebrospinal fluid studies. J Alzheimers Dis 2011;24:175-185.

15 Squitti R, Salustri C: Agents complexing copper as a therapeutic strategy for the treatment of Alzheimer's disease. Curr Alzheimer Res 2009;6:476-487.

16 Ma QF, Li YM, Du JT, Kanazawa K, Nemoto T, Nakanishi H, Zhao YF: Binding of copper (II) ion to an Alzheimer's tau peptide as revealed by MALDI-TOF MS, CD, and NMR. Biopolymers 2005;79:74-85

17 Shumaker SA, Legault C, Kuller L, Rapp SR, Thal L, Lane DS, Fillit H, Stefanick ML, Hendrix SL, Lewis CE, Masaki K, Coker LH; Women's Health Initiative Memory Study: Conjugated equine estrogens and incidence of probable dementia and mild cognitive impairment in postmenopausal women: Women's Health Initiative Memory Study. JAMA 2004;291:2947-2958. 


\section{Case Reports in Neurology}

\begin{tabular}{l|l}
\hline \multicolumn{2}{l}{ Case Rep Neurol 2014;6:181-187 } \\
\hline DOI: 10.1159/000363688 & $\begin{array}{l}\text { C 2014 S. Karger AG, Basel } \\
\text { www.karger.com/crn }\end{array}$ \\
\hline
\end{tabular}

Amtage et al.: Estrogen Intake and Copper Depositions: Implications for Alzheimer's Disease?
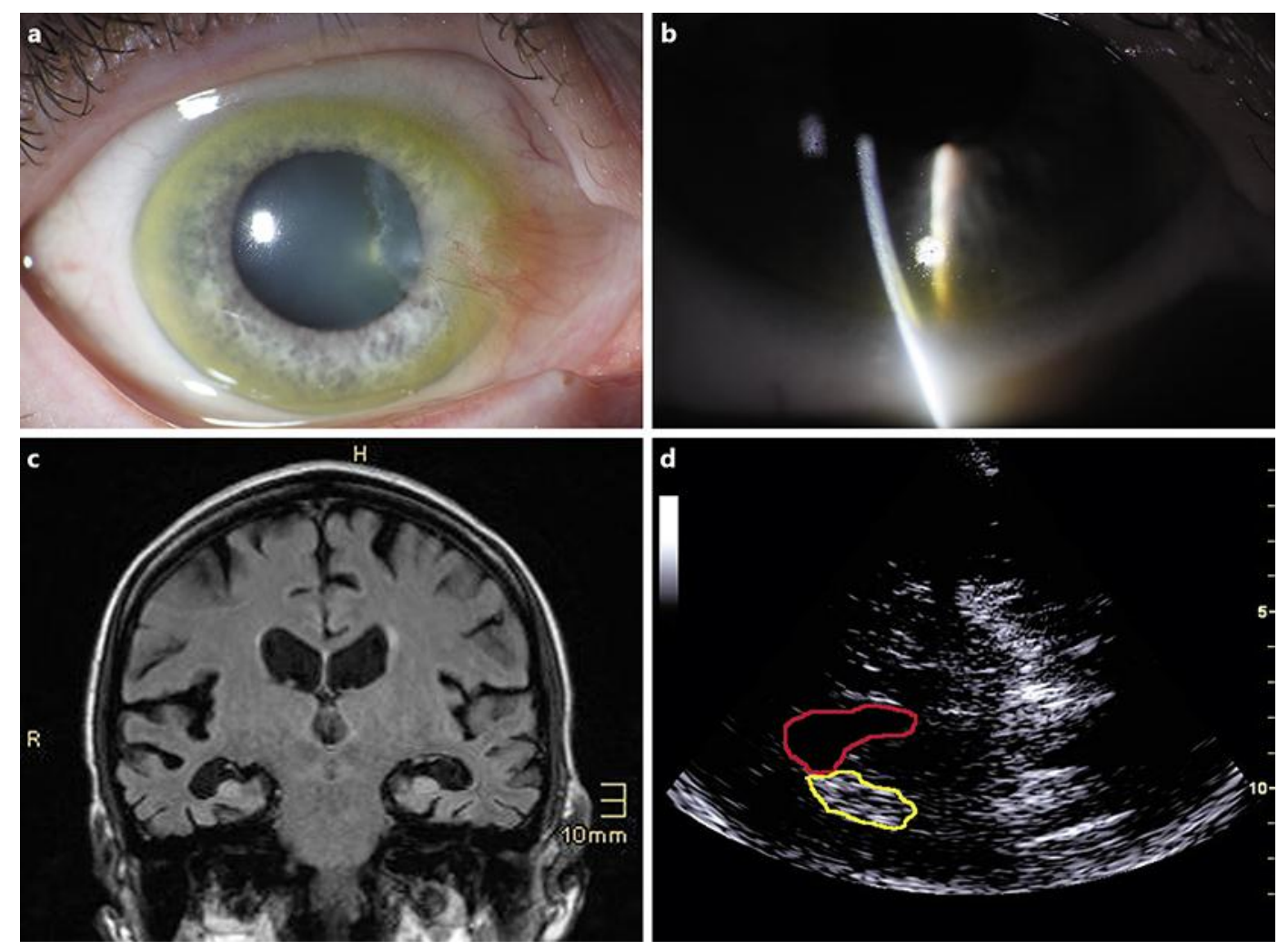

Fig. 1. Ophthalmological findings: macroscopic view (a) and slit-lamp examination (b) revealed copper depositions within the Descemet membrane. c Coronary T1-weighted MRI showed temporoparietalaccentuated brain atrophy. d Sonography of the basal ganglia revealed hyperechogenicity of both lentiform nuclei, demonstrated here for the right lentiform nucleus (yellow) neighboring the right lateral ventricle (red). 
Amtage et al.: Estrogen Intake and Copper Depositions: Implications for Alzheimer's Disease?

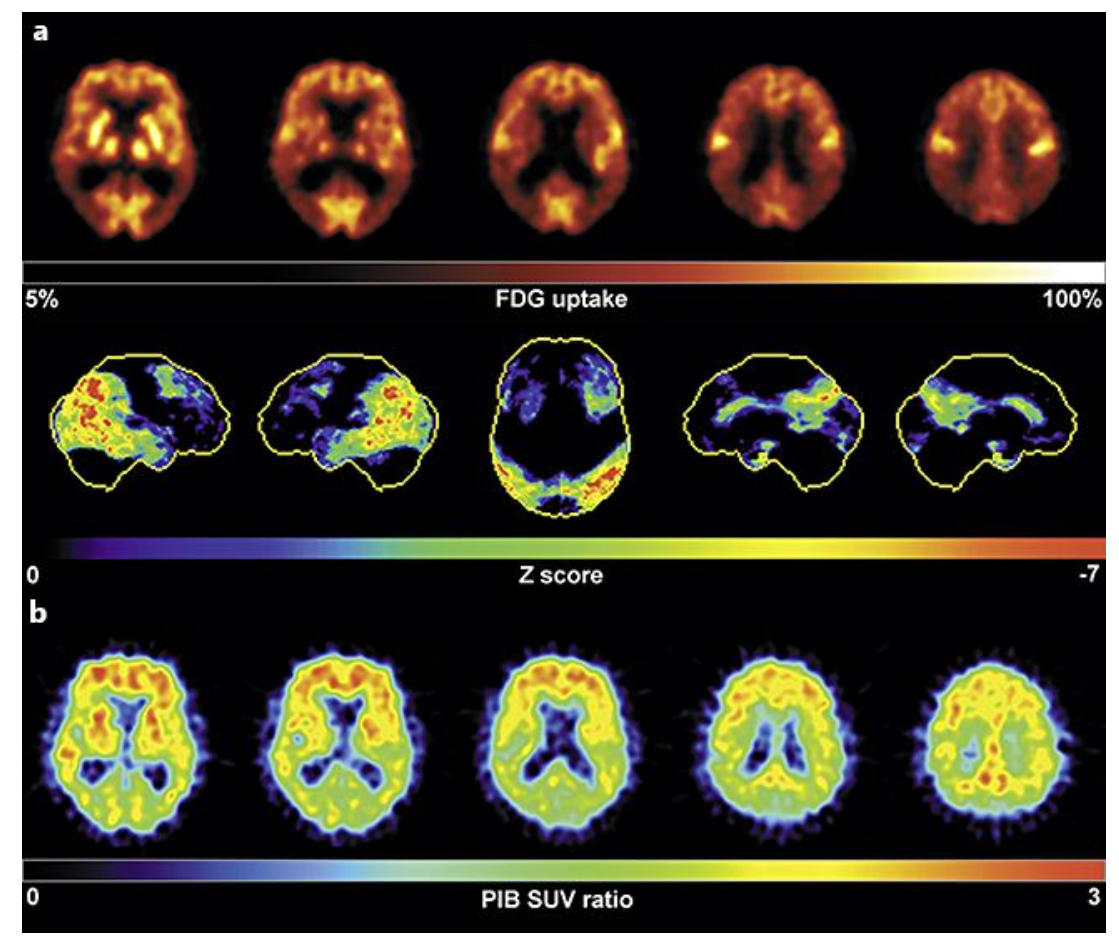

Fig. 2. Results of ${ }^{18 F D G ~(a) ~ a n d ~[11 C] P I B ~(b) ~ P E T ~ e x a m i n a t i o n s . ~ a ~ T h e ~ u p p e r-~ a n d ~ l o w e r-r o w ~ i m a g e s ~ s h o w ~}$ transaxial ${ }^{18}$ FDG PET images and three-dimensional surface projections (left and right lateral views, top view, right and left mesial views) of regions with decreased ${ }^{18 F D G}$ uptake (color-coded as $\mathrm{Z}$ score), respectively. Note the marked bilateral hypometabolism of the temporoparietal region and of the posterior cingulate gyrus/precuneus. A less marked hypometabolism was also found in the bilateral frontal and mesiotemporal lobe. $\mathbf{b}$ Depiction of the corresponding transaxial $\left[{ }^{11} \mathrm{C}\right]$ PIB PET images that show the typical regional pattern of fibrillar amyloid- $\beta$ binding in $\mathrm{AD}$ (strongest in the frontal cortex and posterior cingulate gyrus/precuneus, less so in the temporoparietal cortex). 